\title{
Nanogram per Milligram of Creatinine
}

National Cancer Institute

\section{Source}

National Cancer Institute. Nanogram per Milligram of Creatinine. NCI Thesaurus. Code C85744.

Nanograms per milligram of creatinine. 